\title{
Erratum to: Stable algorithm for event detection in event-driven particle dynamics: logical states
}

\author{
Severin Strobl ${ }^{1}\left[\right.$ Marcus N. Bannerman $^{2} \cdot$ Thorsten Pöschel $^{1}$
}

Published online: 27 April 2016

(C) OWZ 2016

\section{Erratum to: Comp. Part. Mech.}

\section{DOI 10.1007/s40571-016-0106-7}

The original publication of the article contains an error in line number 8 of Algorithm 2. The correct version of the Algorithm 2 is provided in this erratum.

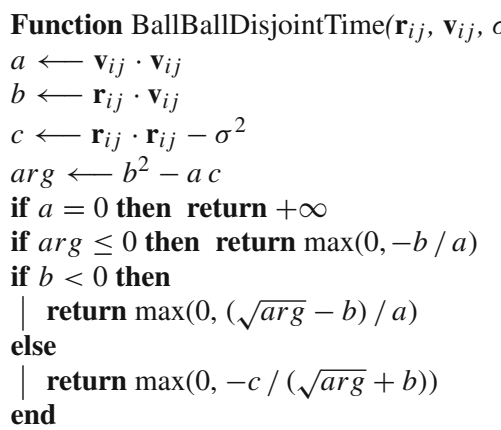

Algorithm 2: A stable algorithm for detecting when two initially intersecting balls become disjoint. This determines the time until $f_{B B} \geq 0$ and $\dot{f}_{B B}>0$ or returns $+\infty$ if this does not occur in the future.

The online version of the original article can be found under doi:10.1007/s40571-016-0106-7.

Severin Strobl

severin.strobl@fau.de

1 Institute for Multiscale Simulation,

Friedrich-Alexander-Universität Erlangen-Nürnberg,

Nägelsbachstr. 49b, 91052 Erlangen, Germany

2 School of Engineering, University of Aberdeen,

Fraser Noble Building, Aberdeen AB24 3UE, UK 